Vietnam Journal of Mechanics, VAST, Vol.33, No. 1 (2011), pp. $41-54$

\title{
CONTINUOUS ELEMENT FOR VIBRATION ANALYSIS OF THICK SHELLS OF REVOLUTION
}

\author{
Nguyen Manh Cuong, Tran Ich Thinh \\ Hanoi University of Technology
}

\begin{abstract}
This paper presents a new numerical method: Continous Element Method (CEM) for vibration analysis of thick shells of revolution taking into account the shear deflection effects. Natural frequencies and harmonic responses of cylindrical and conical shells subjected to different boundary conditions obtained with this kind of formulation are in close agreement with finite element solutions. The main advantage is the reduction of the size of the model thus allows the high precision in the results for a large frequency range.
\end{abstract}

Key words: Continuous element method, Dynamic stiffness matrix, Dynamic transfer matrix, Shell of revolution, Harmonic response.

\section{INTRODUCTION}

The Dynamic Stiffness Method is a highly effcient way to analyse harmonic responses of structures made up of many simple elements. This method, also known as the Continuous Element Method (CEM), is particularly well-suited to pylon and beam lattice structures. It is based on the so-called dynamic stiffness matrix, denoted hereafter $K(\omega)$, which gives exact relations between forces and displacements at the ends of a structural element [1]. The exactness of the relation can only be understood with regard to a given elastodynamic theory. In the case of straight beam assemblies, exact solutions of the equations of motion according to Euler - Bernoulli assumptions or the Rayleigh and Timoshenko theories have been widely used to derive the dynamic stiffness matrices [1], [8]-[11]. During the 1980s, several computer codes were developed based on this method. The calculation of the dynamic stiffness matrix for curved beams is based on an integration of the equations of motion whose associated eigenproblems has been solved beforehand $[12,13]$. Using a very similar approach, an axisymmetric cylindrical shell element with no shear effects has been developed as well [4].

The next stage was the development of plate elements. For this kind of elements, the solution of the equations of motion is theoretically unattainable. Nevertheless, a dynamic stiffness matrix has been built from series expansion [13].

This paper is a direct continuation and a significant advantage of the above mentioned research. Its main object is to present a procedure to obtain the dynamic stiffness matrix of an axisymmetric shell which takes into account both the rotatory inertia and 
shear deformations effects. The method is based on a series expansion of the displacement of the cross-section's middle line and an integration of the dynamic transfer matrix.

\section{STRONG FORMULATION}

Consider one axisymmetric surface with $\mathrm{OZ}$ called right axis of revolution. The most common of this type of surface are of course the cylinder and the cone. The geometry of the axisymmetric shell is shown in Fig. 1.

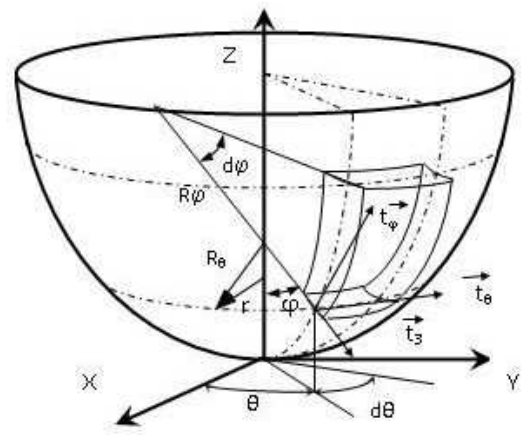

Fig. 1. Geometry of an axisymmetric shell

Fig. 2 describes the displacements, rotations, forces and moments in the normal facet of the axisymmetric shell. They are named: $\mathrm{u}_{\varphi}, u_{\theta}, u_{3}, \beta_{\varphi}, \beta_{\theta}, N_{\varphi \varphi}, N_{\theta \varphi}, N_{\theta \theta}, N_{\varphi \theta}$, $Q_{\varphi 3}, Q_{\theta 3}, M_{\varphi \varphi}, M_{\theta \theta}, M_{\varphi \theta}, M_{\theta \varphi}[3]$.

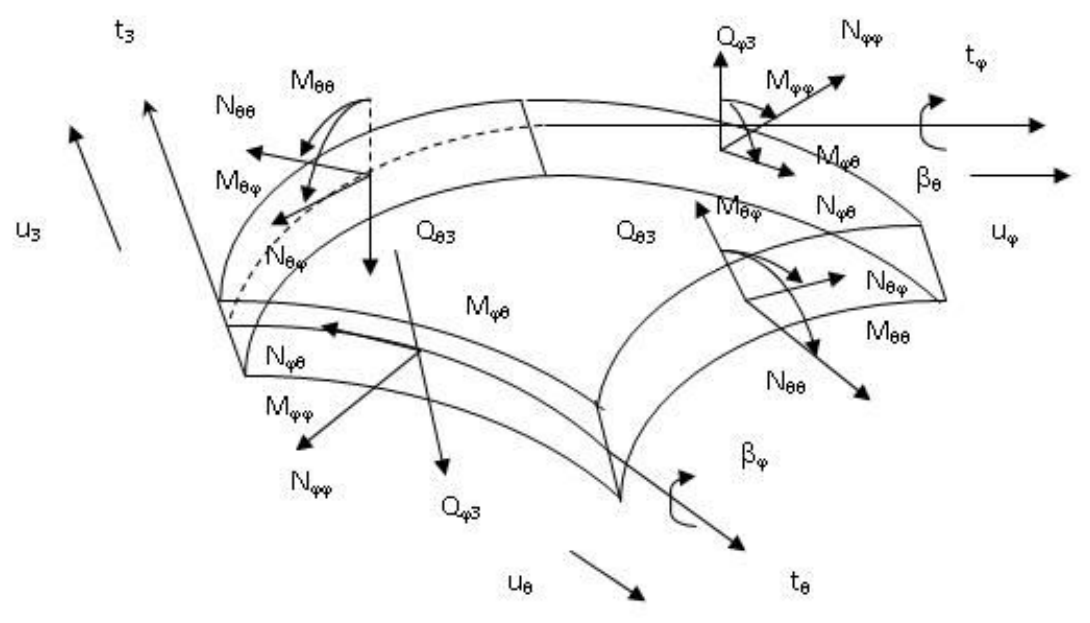

Fig. 2. Axisymmetric shell element 
The deformation-displacements relations and the relations between internal efforts, moments and displacements are written by [3]:

$$
\begin{aligned}
& \varepsilon_{\varphi \varphi}^{0}=\frac{1}{R_{\varphi}}\left(\frac{\partial u_{\varphi}}{\partial \varphi}+u_{3}\right), \\
& \varepsilon_{\theta \theta}^{0}=\frac{1}{R_{\varphi} \sin \varphi} \frac{\partial u_{\theta}}{\partial \theta}+\frac{u_{\varphi}}{R_{\varphi} R_{\theta} \sin \varphi} R_{\varphi} \cos \varphi+\frac{u_{3}}{R_{\theta}} \\
& \varepsilon_{\varphi \theta}^{0}=\frac{R_{\theta} \sin \varphi}{R_{\varphi}} \frac{\partial}{\partial \varphi}\left(\frac{u_{\theta}}{R_{\theta} \sin \varphi}\right)+\frac{1}{R_{\theta} \sin \varphi} \frac{\partial u_{\varphi}}{\partial \theta}, \\
& k_{\varphi \varphi}^{0}=\frac{1}{R_{\varphi}} \frac{\partial \beta_{\varphi}}{\partial \varphi} \\
& k_{\theta \theta}^{0}=\frac{1}{R_{\theta} \sin \varphi} \frac{\partial \beta_{\theta}}{\partial \theta}+\frac{\beta_{\varphi}}{R_{\varphi} R_{\theta} \sin \varphi} R_{\varphi} \cos \varphi, \\
& k_{\varphi \theta}^{0}=\frac{R_{\theta} \sin \varphi}{R_{\varphi}} \frac{\partial}{\partial \varphi}\left(\frac{\beta_{\theta}}{R_{\theta} \sin \varphi}\right)+\frac{1}{R_{\theta} \sin \varphi} \frac{\partial \beta_{\varphi}}{\partial \theta}, \\
& N_{\varphi \varphi}=D\left(\varepsilon_{\varphi \varphi}^{0}+v \varepsilon_{\theta \theta}^{0}\right), N_{\varphi \varphi}=D\left(\varepsilon_{\theta \theta}^{0}+v \varepsilon_{\varphi \varphi}^{0}\right), N_{\varphi \theta}=N_{\theta \varphi}=\frac{D(1-v)}{2} \varepsilon_{\varphi \theta}^{0}, \\
& M_{\varphi \varphi}=K\left(k_{\varphi \varphi}^{0}+v k_{\theta \theta}^{0}\right), M_{\varphi \varphi}=K\left(k_{\theta \theta}^{0}+v k_{\varphi \varphi}^{0}\right), M_{\varphi \theta}=M_{\theta \varphi}=\frac{K(1-v)}{2} k_{\varphi \theta}^{0}, \\
& Q_{\varphi 3}=K^{\prime} G h\left(\frac{1}{R_{\varphi}} \frac{\partial u_{3}}{\partial \varphi}-\frac{u_{\varphi}}{R_{\varphi}}+\beta_{\varphi}\right), Q_{\theta 3}=K^{\prime} G h\left(\frac{1}{R_{\theta} \sin \varphi} \frac{\partial u_{3}}{\partial \theta}-\frac{u_{\theta}}{R_{\theta}}+\beta_{\theta}\right),
\end{aligned}
$$

with: $D=\frac{E h}{1-v^{2}}, K=\frac{E h^{3}}{12\left(1-v^{2}\right)}, K^{\prime}:$ shear correction factor, $h$ : thickness of the shell, $v$ : Poisson's ratio; E: Young modulus. The dynamic equilibrium relationships are written by [2]:

$$
\begin{aligned}
& \frac{\partial\left(N_{\varphi \varphi} R_{\theta} \sin \varphi\right)}{\partial \varphi}+R_{\varphi} \frac{\partial N_{\theta \varphi}}{\partial \theta}-N_{\theta \theta} R_{\varphi} \cos \varphi+R_{\varphi} R_{\theta} \sin \varphi\left(\frac{Q_{\varphi 3}}{R_{\varphi}}+q_{\varphi}-\rho h \frac{\partial^{2} u_{\varphi}}{\partial t^{2}}\right)=0 \\
& \frac{\partial\left(N_{\varphi \theta} R_{\theta} \sin \varphi\right)}{\partial \varphi}+R_{\varphi} \frac{\partial N_{\theta \theta}}{\partial \theta}+N_{\theta \varphi} R_{\varphi} \cos \varphi+R_{\varphi} R_{\theta} \sin \varphi\left(\frac{Q_{\theta 3}}{R_{\theta}}+q_{\theta}-\rho h \frac{\partial^{2} u_{\theta}}{\partial t^{2}}\right)=0 \\
& \frac{\partial\left(Q_{\varphi 3} R_{\theta} \sin \varphi\right)}{\partial \varphi}+R_{\varphi} \frac{\partial Q_{\theta 3}}{\partial \theta}-R_{\varphi} R_{\theta} \sin \varphi\left(\frac{N_{\varphi \varphi}}{R_{\varphi}}+\frac{N_{\theta \theta}}{R_{\theta}}-q_{3}+\rho h \frac{\partial^{2} u_{3}}{\partial t^{2}}\right)=0 \\
& \frac{\partial\left(M_{\varphi \varphi} R_{\theta} \sin \varphi\right)}{\partial \varphi}+R_{\varphi} \frac{\partial M_{\theta \varphi}}{\partial \theta}-M_{\theta \theta} R_{\varphi} \cos \varphi-R_{\varphi} R_{\theta} \sin \varphi\left(Q_{\varphi 3}+\frac{\rho h^{3}}{12} \frac{\partial^{2} \beta_{\varphi}}{\partial t^{2}}\right)=0 \\
& \frac{\partial\left(M_{\varphi \theta} R_{\theta} \sin \varphi\right)}{\partial \varphi}+R_{\varphi} \frac{\partial M_{\theta \theta}}{\partial \theta}-M_{\theta \varphi} R_{\varphi} \cos \varphi-R_{\varphi} R_{\theta} \sin \varphi\left(Q_{\theta 3}+\frac{\rho h^{3}}{12} \frac{\partial^{2} \beta_{\theta}}{\partial t^{2}}\right)=0
\end{aligned}
$$




\section{EXPRESSION OF THE SOLUTION}

As part of the dynamic study of an axisymmetric shell in harmonic vibrations, the temporal terms are separated from spatial terms: $\mathbf{u}(s, \theta, t)=\mathbf{u}(s, \theta) e^{i \omega t}, \mathbf{f}(s, \theta, t)=$ $\mathbf{f}(s, \theta) e^{i \omega t}$ where $\mathbf{u}(s, \theta)$ is generalized displacements and $\mathbf{f}(s, \theta)$ is generalized forces. This leads to a system of partial differential equations [7], involving only the fields $u_{\varphi}, u_{\theta}, u_{3}$, $\beta_{\varphi}, \beta_{\theta}, N_{\varphi \varphi}, N_{\varphi \theta}, Q_{\varphi 3}, M_{\varphi \varphi}, M_{\varphi \theta}$. Other fields: $N_{\theta \theta}, N_{\theta \varphi}, Q_{\theta 3}, M_{\theta \theta}$ and $M_{\theta \varphi}$ are deduced by earlier behavioral relationships (1).

$\left\{y_{m}(s, \omega)\right\}=\left\{u_{\varphi}, u_{\theta}, u_{3}, \beta_{\varphi}, \beta_{\theta}, Q_{\varphi 3}, N_{\varphi \varphi}, N_{\varphi \theta}, M_{\varphi \varphi}, M_{\varphi \theta}\right\}$ is the vector of state solution.

\subsection{Symmetrical vibration, anti-symmetrical vibration}

The solution is expressed by the form of development in Fourier series. It is necessary to consider the symmetrical forms of solutions and anti-symmetrical forms with respect to the variable $\theta$. For symmetric vibration, the symmetrical variables are defined by:

$$
\left(\begin{array}{c}
u_{3}(s, \theta) \\
u_{\varphi}(s, \theta) \\
\beta_{\varphi}(s, \theta) \\
Q_{\varphi 3}(s, \theta) \\
N_{\varphi \varphi}(s, \theta) \\
N_{\theta \theta}(s, \theta) \\
M_{\varphi \varphi}(s, \theta) \\
M_{\theta \theta}(s, \theta)
\end{array}\right)=\sum_{m=0}^{\infty}\left(\begin{array}{c}
u_{3 m}(s) \\
u_{\varphi m}(s) \\
\beta_{\varphi m}(s) \\
Q_{\varphi 3 m}(s) \\
N_{\varphi \varphi m}(s) \\
N_{\theta \theta m}(s) \\
M_{\varphi \varphi m}(s) \\
M_{\theta \theta m}(s)
\end{array}\right) \cos m \theta,\left(\begin{array}{c}
u_{\theta}(s, \theta) \\
\beta_{\theta}(s, \theta) \\
N_{\varphi \theta}(s, \theta) \\
Q_{\theta 3}(s, \theta) \\
M_{\varphi \theta}(s, \theta)
\end{array}\right)=\sum_{m=0}^{\infty}\left(\begin{array}{c}
u_{\theta m}(s) \\
\beta_{\theta m}(s) \\
N_{\varphi \theta m}(s) \\
Q_{\theta 3 m}(s) \\
M_{\varphi \theta m}(s)
\end{array}\right) \sin m \theta
$$

It is simple to build the solutions for anti-symmetrical vibrations by swapping $\sin (m \theta)$ and $\cos (m \theta)$. The special characteristic of axisymmetric shells is the decoupling of solutions of the problem (4), that means the final solution will be obtained by superimposing the solutions of each of $\mathrm{m}$ modes. To reduce the system, it is more convenient to use the distance s measured along the meridian of the shell than using the angular coordinate $\varphi$. Note that: $\frac{1}{R_{\varphi}} \frac{\partial}{\partial \varphi}=\frac{\partial}{\partial s}$.

The derivatives of state solution with respect to the curvilinear abscissa s are calculated from (1) and (2):

$$
\begin{aligned}
\frac{d u_{\varphi m}}{d s} & =\frac{1}{D} N_{\varphi \varphi m}-\frac{1}{R_{\varphi}} u_{3 m}-v\left(\frac{m}{r} u_{\theta m}+\frac{\cos \varphi}{r} u_{\varphi m}+\frac{\sin \varphi}{r} u_{3 m}\right), \\
\frac{d u_{\theta}}{d s}= & \frac{2 N_{\varphi \theta m}}{D(1-v)}+\frac{\cos \varphi}{r} u_{\theta m}+\frac{m}{r} u_{\varphi m}, \frac{d u_{3 m}}{d s}=\frac{Q_{\varphi 3 m}}{K^{\prime} G h}+\frac{u_{\varphi m}}{R_{\varphi}}-\beta_{\varphi m}, \\
\frac{d \beta_{\varphi m}}{d s} & =\frac{1}{K} M_{\varphi \varphi m}-v\left(\frac{m}{r} \beta_{\theta m}+\frac{\cos \varphi}{r} \beta_{\varphi m}\right), \frac{d \beta_{\theta m}}{d s}=\frac{2 M_{\varphi \theta m}}{K(1-v)}+\frac{\cos \varphi}{r} \beta_{\theta m}+\frac{m}{r} \beta_{\varphi m}, \\
\frac{d N_{\varphi \varphi m}}{d s} & =\left[(v-1) N_{\varphi \varphi m}+D\left(1-v^{2}\right)\left(\frac{m}{r} u_{\theta m}+\frac{\cos \varphi}{r} u_{\varphi m}+\frac{\sin \varphi}{r} u_{3 m}\right)\right] \frac{\cos \varphi}{r} \\
& -\frac{m}{r} N_{\varphi \theta m}-\frac{1}{R_{\varphi}} Q_{\varphi 3 N_{\varphi \theta m}}-q_{\varphi m}-\rho h \omega^{2} u_{\varphi m},
\end{aligned}
$$




$$
\begin{aligned}
\frac{d N_{\varphi \theta m}}{d s} & =-2 N_{\varphi \theta m} \frac{\cos \varphi}{r}-\frac{1}{r}\left[-v m N_{\varphi \varphi m}+D\left(1-v^{2}\right)\left(\frac{-m^{2}}{r} u_{\theta m}-\frac{m \cos \varphi}{r} u_{\varphi m}\right.\right. \\
- & \left.\left.\frac{m \sin \varphi}{r} u_{3 m}\right)\right]-\frac{\sin \varphi}{r} K^{\prime} G h\left(-\frac{m}{r} u_{3 m}-\frac{\sin \varphi}{r} u_{\theta m}+\beta_{\theta m}\right)-q_{\theta m}-\rho h \omega^{2} u_{\theta m} \\
\frac{d Q_{\varphi 3 m}}{d s} & =-\frac{\cos \varphi}{r} Q_{\varphi 3 m}-\frac{1}{r} K^{\prime} G h\left(-\frac{m^{2}}{r} u_{3 m}-\frac{m \sin \varphi}{r} u_{\theta m}+m \beta_{\theta m}\right)+\frac{1}{R_{\varphi}} N_{\varphi \varphi m} \\
& +\frac{\sin \varphi}{r}\left[v N_{\varphi \varphi m}+D\left(1-v^{2}\right)\left(\frac{m}{r} u_{\theta m}+\frac{\cos \varphi}{r} u_{\varphi m}+\frac{\sin \varphi}{r} u_{3 m}\right)\right]-q_{3 m}-\rho h \omega^{2} u_{3 m}, \\
\frac{d M_{\varphi \varphi m}}{d s} & =\frac{\cos \varphi}{r}\left[(v-1) M_{\varphi \varphi m}+K\left(1-v^{2}\right)\left(\frac{m}{r} \beta_{\theta m}+\frac{\cos \varphi}{r} \beta_{\varphi m}\right)\right]-\frac{m}{r} M_{\varphi \theta m} \\
& +Q_{\varphi 3 m}-\frac{\rho h^{3}}{12} \omega^{2} \beta_{\varphi m}, \\
\frac{d M_{\varphi \theta m}}{d s} & =-2 M_{\varphi \theta m} \frac{\cos \varphi}{r}-\frac{1}{r}\left[-v m M_{\varphi \varphi m}+K\left(1-v^{2}\right)\left(-\frac{m^{2}}{r} \beta_{\theta m}-\frac{m \cos \varphi}{r} \beta_{\varphi m}\right)\right] \\
& +K^{\prime} G h\left(-\frac{m}{r} u_{3 m}-\frac{\sin \varphi}{r} u_{\theta m}+\beta_{\theta m}\right)-\frac{\rho h^{3}}{12} \omega^{2} \beta_{\theta m},
\end{aligned}
$$

The system (3) is written in the matrix form:

$$
\frac{d\left\{y_{m}(s, \omega)\right\}}{d s}=\left[A_{m}(s, \omega)\right]\left\{y_{m}(s, \omega)\right\},
$$

This equation will be used to build a dynamic stiffness matrix $\left[K_{m}(\omega)\right]$ which connects the state variables defined by boundary conditions in the abscissa $s=0$ and in the abscissa $s=L$.

\subsection{Dynamic transfer matrix}

The matrix $\left[T_{m}\left(s \rightarrow s^{\prime}, \omega\right)\right]$ is called dynamic transfer matrix. It computes the state solution vector $\left(y_{m}\left(s^{\prime}, \omega\right)\right)$ in $s^{\prime}$ from the state solution vector $\left(y_{m}(s, \omega)\right)$ in $s:\left[T_{m}(s, \omega)\right]=$ $\left[T_{m}(0 \rightarrow s, \omega)\right]$.

So: $\left\{y_{m}(s, \omega)\right\}=\left[T_{m}(s, \omega)\right]\left\{y_{m}(0, \omega)\right\}$,

Therefore, equation (4) will be simplified as follows:

$$
\frac{d\left[T_{m}(s, \omega)\right]}{d s}=\left[A_{m}(s, \omega)\right]\left[T_{m}(s, \omega)\right],
$$

To determine the dynamic transfer matrix $\left[T_{m}(s, \omega)\right]$, the first order equation (5) must be solved. Here, the necessary boundary conditions for the resolution are expressed by: $\left[T_{m}(0, \omega)\right]=[I]$.

\subsection{Expression of dynamic transfer matrix for axisymmetric shells}

The solution of equation (5) is

$$
\left[T_{m}(s, \omega)\right]=e^{\int^{S} A_{m}(l, \omega) d l},
$$


The dynamic transfer matrix $\left[T_{m}(\omega)\right]$ connects the state vector at $s=0$ to the state vector at $s=L$ ( $L$ is the length of shell). Thus, one has

$$
\left[T_{m}(\omega)\right]=e^{\int^{L} A_{m}(s, \omega) d s}=\left[Q_{m}(\omega)\right]\left[\begin{array}{lll}
e^{\lambda_{m 1}(\omega) L} & 0 \\
& \cdots \cdots & \\
0 & & e^{\lambda_{n m}(\omega) L}
\end{array}\right]\left[Q_{m}(\omega)\right]^{-1},
$$

where: $\lambda_{i m}(\omega)$ are eigenvalues of $\int_{0}^{L} A_{m}(s, \omega) d s,\left[Q_{m}(\omega)\right]$ is the matrix of eigenvectors of $\int_{0}^{L} A_{m}(s, \omega) d s$.

\subsection{Expression of dynamic transfer matrix for typical shells}

In case of cylindrical shell, the dynamic transfer matrix is computed by

$$
\left[T_{m}(\omega)\right]=e^{\left[A_{m}(\omega)\right] L}=\left[Q_{m}(\omega)\right]\left[\begin{array}{lll}
e^{\lambda_{1 m}(\omega) L} & 0 \\
& \cdots \cdots & e^{\lambda_{n m}(\omega) L}
\end{array}\right]\left[Q_{m}(\omega)\right]^{-1}
$$

For the conical shell, the dynamic transfer matrix is written:

$$
\left[T_{m}(\omega)\right]=e^{\left[\hat{A}_{m}(\omega)\right] L}=\left[\hat{Q}_{m}(\omega)\right]\left[\begin{array}{ccc}
e^{\hat{\lambda}_{1 m}(\omega) L} & 0 \\
& \cdots \cdots . . . & \\
0 & & e^{\hat{\lambda}_{n m}(\omega) L}
\end{array}\right]\left[\hat{Q}_{m}(\omega)\right]^{-1}
$$

with: $\left[\hat{A}_{m}(\omega)\right]=\frac{1}{L} \int_{0}^{L}\left[A_{m}(\omega, s)\right] d s$,

and: $\hat{\lambda}_{i m}(\omega)$ - eigenvalues of $\hat{A}_{m}(\omega),\left[\hat{Q}_{m}(\omega)\right]$ - matrix of eigenvectors of $\hat{A}_{m}(\omega)$.

\section{DYNAMIC STIFFNESS MATRIX $\left[K_{m}(\omega)\right]$}

\subsection{Definition of the dynamic stiffness matrix}

It links the displacement vector $\left\{U_{m}(0), U_{m}(L)\right\}^{T}$ at the ends of a shell of revolution to the vectors of external force $\left\{F_{\text {mext }}(0), F_{\text {mext }}(L)\right\}^{T}$ at the ends of a shell of revolution. $\left[K_{m}(\omega)\right]$ depends on the pulsation $\omega$ :

$$
\left\{\begin{array}{l}
\left\{F_{\text {mext }}(0)\right\} \\
\left\{F_{\text {mext }}(L)\right\}
\end{array}\right\}=\left[K_{m}(\omega)\right]\left\{\begin{array}{l}
\left\{U_{m}(0)\right\} \\
\left\{U_{m}(L)\right\}
\end{array}\right\} .
$$

\subsection{Expression of the dynamic stiffness matrix $\left[K_{m}(\omega)\right]$}

The dynamic transfer matrix $\left[T_{m}(\omega)\right]$ is decomposed in 4 blocks:

$$
\left[T_{m}(\omega)\right]=\left[\begin{array}{ll}
T_{11}(\omega) & T_{12}(\omega) \\
T_{21}(\omega) & T_{22}(\omega)
\end{array}\right]
$$

and the state vectors at $s=0$ and $s=L$ are: $\left\{F_{m}(0)\right\}=\left\{-F_{\text {mext }}(0)\right\},\left\{F_{m}(L)\right\}=$ $\left\{-F_{\text {mext }}(L)\right\}$. 
Thus, $\left[K_{m}(\omega)\right]$ is expressed by:

$$
\left[K_{m}(\omega)\right]=\left[\begin{array}{ll}
-T_{12}^{-1}(\omega) T_{11}(\omega) & T_{12}^{-1}(\omega) \\
T_{21}(\omega)-T_{22}(\omega) T_{12}^{-1}(\omega) T_{11}(\omega) & T_{22}(\omega) T_{12}^{-1}(\omega)
\end{array}\right]
$$

\section{NUMERICAL RESULTS}

\subsection{Continuous element for circular cylindrical shells}

In this case, $R=\infty ; R_{\theta}=a$, (3) becomes:

$$
\begin{aligned}
\frac{d u_{x m}}{d x} & =\frac{1}{D} N_{x x m}-\frac{v}{a}\left(m u_{\theta m}+u_{3 m}\right), \frac{d u_{\theta m}}{d x}=\frac{2}{D(1-v)} N_{x \theta m}+\frac{m}{a} u_{x m}, \\
\frac{d u_{3 m}}{d x}= & \frac{1}{K^{\prime} G h} Q_{x 3 m}-\beta_{x m}, \\
\frac{d \beta_{x m}}{d x}= & \frac{1}{K} M_{x x m}-\frac{v m}{a} \beta_{\theta m}, \frac{d N_{x x m}}{d x}=-\frac{m}{a} N_{x \theta m}-\rho h \omega^{2} u_{x m}, \\
\frac{d N_{x \theta m}}{d x} & =-\frac{1}{a}\left[-v m N_{x x m}-\frac{D}{a}\left(v^{2}-1\right)\left(-m^{2} u_{\theta m}-m u_{3 m}\right)\right] \\
& -\frac{K^{\prime} G h}{a}\left(-\frac{m}{a} u_{3 m}-\frac{1}{a} u_{\theta m}+\beta_{\theta m}\right)-\rho h \omega^{2} u_{\theta m}, \\
\frac{d Q_{x 3 m}}{d x} & =-\frac{K^{\prime} G h}{a}\left(-\frac{m^{2}}{a} u_{3 m}-\frac{m}{a} u_{\theta m}+m \beta_{\theta m}\right) \\
& +\frac{1}{a}\left[v N_{x x m}-\frac{D}{a}\left(v^{2}-1\right)\left(m u_{\theta m}+u_{3 m}\right)\right]-\rho h \omega^{2} u_{3 m}, \\
\frac{d M_{x x m}}{d x} & =-\frac{m}{a} M_{x \theta m}+Q_{x 3 m}-\frac{\rho h^{3} \omega^{2}}{12} \beta_{x m}, \\
\frac{d M_{x \theta m}}{d x} & =-\frac{1}{a}\left[-v m M_{x x m}+\frac{K}{a} m^{2}\left(v^{2}-1\right) \beta_{\theta m}\right] \\
& +K^{\prime} G h\left(-\frac{m}{a} u_{3 m}-\frac{1}{a} u_{\theta m}+\beta_{\theta m}\right)-\frac{\rho h^{3} \omega^{2}}{12} \beta_{\theta m},
\end{aligned}
$$

The continuous element for cylindrical shells is constructed using these equations.

The numerical calculation is performed with: $E=2 x 10^{10} \mathrm{~N} / \mathrm{m}^{2}, \rho=7800 \mathrm{~kg} / \mathrm{m}^{3}$, $\nu=0.3, K^{\prime}=2 / 3$.

\section{a. Frequencies of a clamped-clamped cylindrical shell}

Table 1. Comparison of reduced pulse $\Psi=\omega R \sqrt{\frac{\left(1-v^{2}\right) \rho}{E}}$ for an isotropic clamped-clamped cylindrical shell $(m=1, n=1, \nu=0.3, H / L=0.1)$

\begin{tabular}{|c|c|c|c|}
\hline \multicolumn{4}{|c|}{$H / L=0.1$} \\
\hline$H / R$ & Finite Elements 3D (Loy \& Lam) $[6]$ & Loy \& Lam $[6]$ & Continuous Element \\
\hline 0.2 & 0.6684 & 0.69319 & 0.6502 \\
\hline 0.4 & 0.3502 & 0.36463 & 0.3419 \\
\hline
\end{tabular}


Table 2. Comparison of reduced pulse $\Psi=\omega R \sqrt{\frac{\left(1-v^{2}\right) \rho}{E}}$ for an isotropic clamped-clamped cylindrical shell $(m=1, n=1, \nu=0.3, H / L=0.2)$

\begin{tabular}{|c|c|c|c|}
\hline \multicolumn{4}{|c|}{$H / L=0.2$} \\
\hline$H / R$ & Finite Elements 3D (Loy \& Lam) $[6]$ & Loy \& Lam [6] & Continuous Element \\
\hline 0.2 & 1.3583 & 1.39567 & 1.3328 \\
\hline 0.4 & 0.7739 & 0.79219 & 0.7692 \\
\hline
\end{tabular}

Table 3. Comparison of reduced pulse $\Psi=\omega R \sqrt{\frac{\left(1-v^{2}\right) \rho}{E}}$ for an isotropic clamped-clamped cylindrical shell $(m=1, n=1, \nu=0.3, H / L=0.4)$

\begin{tabular}{|c|c|c|c|}
\hline \multicolumn{4}{|c|}{$H / L=0.4$} \\
\hline$H / R$ & Finite Elements 3D (Loy \& Lam) $[6]$ & Loy \& Lam $[6]$ & Continuous Element \\
\hline 0.2 & 3.4464 & 3.27162 & 3.4402 \\
\hline 0.4 & 1.7186 & 1.74212 & 1.6981 \\
\hline
\end{tabular}

First, CEM formulation is compared to a Finite Element 3D model and to another analytical model developped by Lam [6] (Table 1, Table 2, Talbe 3) for an isotropic clamped-clamped cylindrical shell with different values of $\mathrm{H} / \mathrm{L}(0.1,0.2,0.4)$.

Table 4. Comparison of reduced pulse $\Omega=\frac{\omega H}{\pi} \sqrt{\frac{\rho}{G}}$ for an isotropic free - free cylindrical shell $(m=1, n=1, \nu=0.3, H / L=0.2)$

\begin{tabular}{|c|c|c|}
\cline { 2 - 3 } \multicolumn{1}{c|}{} & \multicolumn{2}{c|}{$H / R$} \\
\cline { 2 - 3 } \multicolumn{1}{c|}{} & 0.1 & 0.2 \\
\hline Finite Elements 3D (Loy \& Lam) [6] & 0.07724 & 0.14860 \\
\hline Loy \& Lam [6] & 0.07618 & 0.10704 \\
\hline Continuous Element & 0.07541 & 0.10273 \\
\hline
\end{tabular}

Then, reduced pulse of an isotropic free-free cylindrical shell is also investigated in Table 4. Results obtained by these comparisons are excelents which validate our CEM model.

\section{b. Frequencies of a clamped-free cylindrical shell}

The properties of the cylindrical shell are: $R=0.3 \mathrm{~m}, L=0.4 \mathrm{~m}, h=0.002 \mathrm{~m}$, $E=2.1 x 10^{11} \mathrm{~N} / \mathrm{m}^{2}, \rho=7800 \mathrm{~kg} / \mathrm{m}^{3}, \nu=0.3$.

The Finite Element model used is a shell model with inclusion of transverse shear which consists of $180 \times 6$ mesh elements (Fig.3) meanwhile only one element is sufficient for the calculation by the Continous Element method.

Table 5. presents the comparison of natural frequencies (in hertz) of the clampedfree cylindical shell by using 3 methods: our formulation, results of CEM by Le Sourne [4] and thoses obtained by Finite Element Method (Ansys). The obtained results are very satisfactory. These answers are very close because the same formulation (taking into account the transverse shear) is used. The advantage of the method of continuous elements 

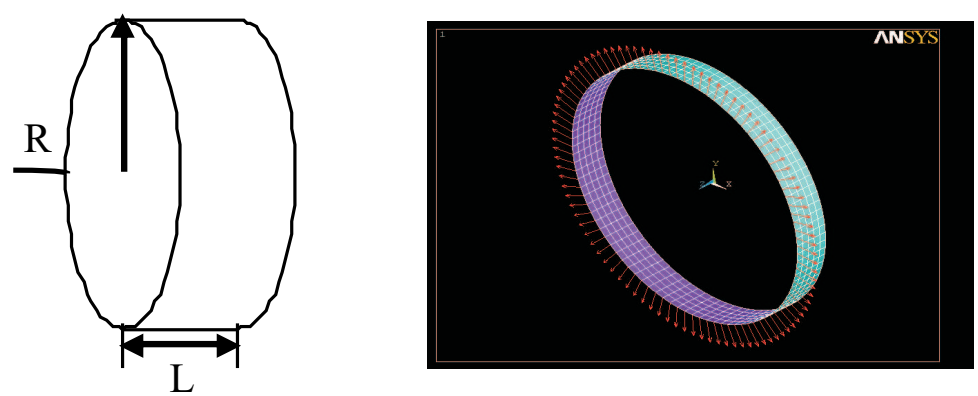

Fig. 3. Mesh by Continuous Element (Left) and by Finite Element (Right) model

Table 5. Comparison of natural frequencies (in hertz) of the clamped-free cylindical shell

\begin{tabular}{|c|c|c|c|}
\hline Mode & Continuous Element & Le Sourne [4] & Finite Element \\
\hline 1 & 234.64 & 234.25 & 234.87 \\
\hline 3 & 241.58 & 241.97 & 242.02 \\
\hline 5 & 289.14 & 288.10 & 289.29 \\
\hline 7 & 289.68 & 289.97 & 290.52 \\
\hline 9 & 362.68 & 362.79 & 364.14 \\
\hline 11 & 427.06 & 425.39 & 427.19 \\
\hline 13 & 452.57 & 452.18 & 454.97 \\
\hline 15 & 556.24 & 554.94 & 559.89 \\
\hline 17 & 602.81 & $585.50^{*}$ & 604.61 \\
\hline 19 & 624.19 & 615.50 & 626.71 \\
\hline 21 & 627.88 & $602.70^{*}$ & 629.35 \\
\hline 23 & 672.38 & $669.80^{*}$ & 677.77 \\
\hline 25 & 682.60 & 681.52 & 686.15 \\
\hline 27 & 700.69 & 698.26 & 700.80 \\
\hline
\end{tabular}

is the reduced computation time, a minimum storage of data and a mesh much easier since reduced to a single element. Several terms of $m$ are enough for obtaining a solution with high precision. Differences appear between the two models beyond $2000 \mathrm{~Hz}$. By increasing the fineness of the mesh, the responses obtained by the Finite Element method converge to that obtained by our formulation, it is noted also that the results of Finite Element model will degrade quickly when going up in frequencies.

\subsection{Continuous element for circular conical shells}

Here: $R_{\varphi}=0, R_{\theta}=x \tan \alpha, R_{\theta} \sin \varphi=x \sin \alpha, R_{\varphi} d \varphi=d x,(3)$ is written as:

$$
\begin{aligned}
\frac{d u_{3 m}}{\partial x} & =\frac{1}{K^{\prime} G h} Q_{x 3 m}-\beta_{x m}, \\
\frac{d \beta_{\theta m}}{\partial x} & =\frac{2}{K(1-v)} M_{x \theta m}+\frac{1}{x} \beta_{\theta m}+\frac{m}{x \sin \alpha} \beta_{x m},
\end{aligned}
$$




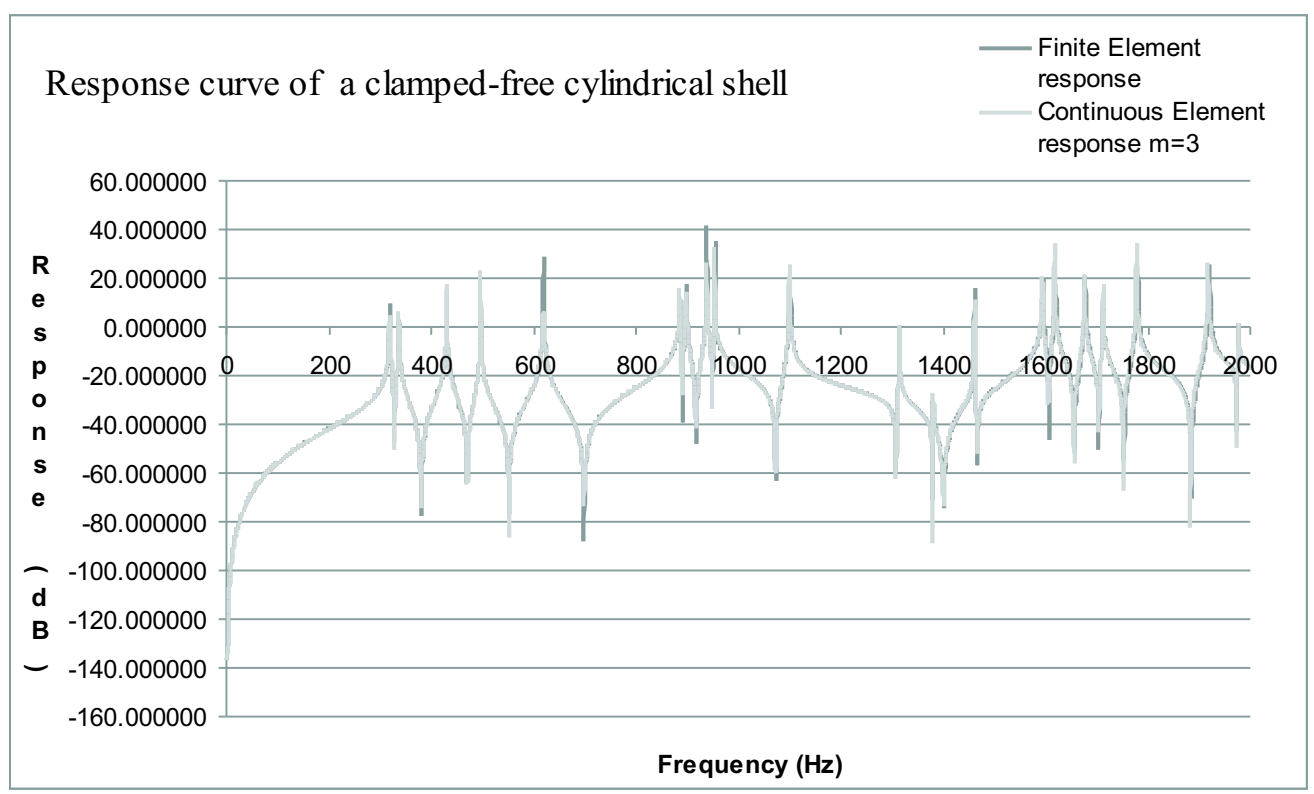

Fig. 4. Comparison of frequency response of a clamped-free cylindrical shell obtained by finite element and continuous elements methods

$$
\begin{aligned}
& \frac{d u_{\theta m}}{\partial x}=\frac{2}{D(1-v)} N_{x \theta m}+\frac{1}{x} u_{\theta m}+\frac{m}{x \sin \alpha} u_{x m}, \\
& \frac{d N_{x x m}}{\partial x}=-\rho h \omega^{2} u_{x m}-\frac{m}{x \sin \alpha} N_{x \theta m}-\frac{1}{x}\left[(1-v) N_{x x m}-D\left(1-v^{2}\right)\right. \\
& \left.\left(\frac{m}{x \sin \alpha} u_{\theta m}+\frac{1}{x} u_{x m}+\frac{1}{x \tan \alpha} u_{3 m}\right)\right] \text {, } \\
& \frac{d N_{x \theta m}}{\partial x}=-\frac{2}{x} N_{x \theta m}-\frac{1}{x \sin \alpha}\left[-v m N_{x x m}+D\left(1-v^{2}\right)\left(\frac{-m^{2}}{x \sin \alpha} u_{\theta m}-\frac{m}{x} u_{x m}\right.\right. \\
& \left.\left.-\frac{m}{x \tan \alpha} u_{3 m}\right)\right]-\frac{K^{\prime} G h}{x \tan \alpha}\left(\frac{-m}{x \sin \alpha} u_{3 m}-\frac{1}{x \tan \alpha} u_{\theta m}+\beta_{\theta m}\right)-\rho h \omega^{2} u_{\theta m}, \\
& \frac{d Q_{x 3 m}}{\partial x}=-\frac{1}{x} Q_{x 3 m}-\frac{K^{\prime} G h}{x \sin \alpha}\left(\frac{-m^{2}}{x \sin \alpha} u_{3 m}-\frac{m}{x \tan \alpha} u_{\theta m}+m \beta_{\theta m}\right) \\
& +\frac{1}{x \tan \alpha}\left[v N_{x x m}+D\left(1-v^{2}\right)\left(\frac{m}{x \sin \alpha} u_{\theta m}+\frac{1}{x} u_{x m}+\frac{1}{x \tan \alpha} u_{3 m}\right)\right]-\rho h \omega^{2} u_{3 m}, \\
& \frac{d M_{x x m}}{\partial x}=-\frac{\rho h^{3} \omega^{2}}{12} \beta_{x m}-\frac{m}{x \sin \alpha} M_{x \theta m}+Q_{x 3 m}-\frac{1}{x}\left[(1-v) M_{x x m}\right. \\
& \left.-K\left(1-v^{2}\right)\left(\frac{m}{x \sin \alpha} \beta_{\theta m}+\frac{1}{x} \beta_{x m}\right)\right],
\end{aligned}
$$




$$
\begin{aligned}
& \frac{d M_{x \theta}}{\partial x}=-\frac{2}{x} M_{x \theta m}-\frac{1}{x \sin \alpha}\left[-m v M_{x x m}+K\left(1-v^{2}\right)\left(\frac{-m^{2}}{x \sin \alpha} \beta_{\theta m}-\frac{m}{x} \beta_{x m}\right)\right] \\
& -K^{\prime} G h\left(-\frac{m}{x \sin \alpha} u_{3 m}-\frac{1}{x \tan \alpha} u_{\theta m}+\beta_{\theta m}\right)+\frac{\rho h^{3} \omega^{2}}{12} \beta_{\theta m}, \\
& \frac{d u_{x m}}{\partial x}=\frac{1}{D} N_{x x m}-v\left(\frac{m}{x \sin \alpha} u_{\theta m}+\frac{1}{x} u_{x m}+\frac{1}{x \tan \alpha} u_{3 m}\right), \\
& \frac{d \beta_{x m}}{\partial x}=\frac{1}{K} M_{x x m}-v\left(\frac{m}{x \sin \alpha} \beta_{\theta m}+\frac{1}{x} \beta_{x m}\right),
\end{aligned}
$$

\section{a. Frequencies of a clamped-clamped conical shell}

Consider a free conical shell with the following characteristics:

$$
R_{1}=0.4 \mathrm{~m}, L=0.1 \mathrm{~m}, h=0.002 \mathrm{~m}, \alpha=30^{\circ}, E=2.1 \times 10^{11} \mathrm{~N} / \mathrm{m}^{2}, \rho=7800 \mathrm{~kg} / \mathrm{m}^{3}, \nu=0.3 \text {. }
$$

The Finite Element model used is a shell model with inclusion of transverse shear which consists of $180 \times 6$ and $210 \times 6$ mesh elements (Fig. 5) meanwhile only one element is sufficient for the the Continous Element model.
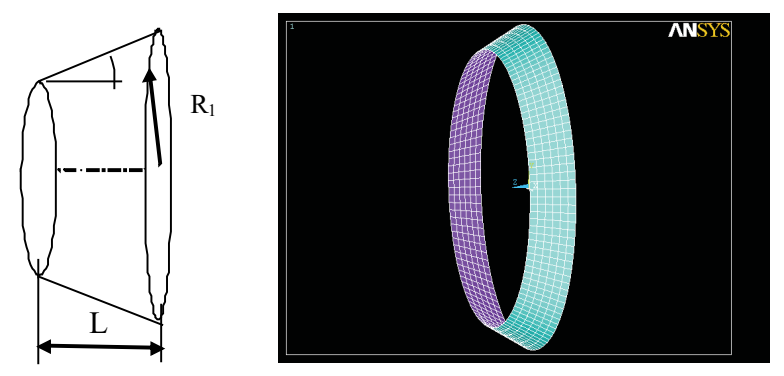

Fig. 5. Mesh by Continuous Element (Left) and Finite Element (Right) method

Table 6. Comparison of reduced pulse $\lambda$ of a free conical shell by various approaches

\begin{tabular}{|c|c|c|c|}
\hline Mode & Continous Element & Le Sourne & Finite Element \\
\hline 1 & 9.57 & 9.8 & 9.5850 \\
\hline 3 & 26.71 & 27.2 & 26.980 \\
\hline 5 & 27.69 & 27.8 & 27.801 \\
\hline 7 & 51.43 & 51.8 & 51.653 \\
\hline 9 & 83.35 & 83.6 & 83.500 \\
\hline 11 & 89.80 & 91.6 & 89.832 \\
\hline 13 & 122.09 & 122.0 & 122.51 \\
\hline 15 & 162.10 & 164.3 & 162.20 \\
\hline 17 & 168.07 & 167.0 & 168.69 \\
\hline 19 & 221.05 & 221.5 & 222.07 \\
\hline 21 & 231.76 & 234.6 & 231.97 \\
\hline 23 & 281.11 & 281.5 & 282.68 \\
\hline 25 & 299.86 & 302.0 & 300.28 \\
\hline 27 & 348.18 & 348.1 & 350.56 \\
\hline 29 & 369.34 & 370.0 & 370.01 \\
\hline 31 & 422.30 & 422.3 & 425.77 \\
\hline 33 & 442.07 & 442.5 & 443.14 \\
\hline 35 & 503.52 & 502.8 & 508.36 \\
\hline
\end{tabular}


Table 6. shows results computed by different methods: our formulation, those of Le Sourne [4] and Finite Element model. CEM model is closer to Finite Element model when the meshing is increased

\section{b. Frequencies of a free-free conical shell}

The calculation is done with the following data: $E=2 \times 10^{10} \mathrm{~N} / \mathrm{m}^{2}, \rho=7800 \mathrm{~kg} / \mathrm{m}^{3}$, $\nu=0.3, K^{\prime}=2 / 3$. The reduced pulse is calculated by: $\lambda=\omega \frac{\left(R-\frac{\operatorname{Ltg} \alpha}{2}\right)}{\sin \alpha} \sqrt{\frac{\rho(1-v)}{E}}$

Table 7. Comparison of the angular frequencies of conical shells by different methods

\begin{tabular}{|c|c|c|c|c|c|c|c|}
\hline & & & & \multicolumn{2}{|c|}{ Mindlin theory } & \multicolumn{2}{c|}{ Classical theory } \\
\hline$\alpha$ & $h / R$ & $L / R$ & $\begin{array}{c}\text { Continuous } \\
\text { Element }\end{array}$ & $\begin{array}{c}\text { Formulation } \\
\text { of Naghdi }\end{array}$ & $\begin{array}{c}\text { Formulation } \\
\text { of Love }\end{array}$ & $\begin{array}{c}\text { Formulation } \\
\text { of Naghdi }\end{array}$ & $\begin{array}{c}\text { Formulation } \\
\text { of Love }\end{array}$ \\
\hline & & & $\sqrt{\lambda_{C E}}$ & $\sqrt{\lambda_{\text {Mindlin }}}$ & $\sqrt{\lambda_{\text {Mindlin }}}$ & $\sqrt{\lambda_{\text {Classical }}}$ & $\sqrt{\lambda_{\text {Classical }}}$ \\
\hline $5^{\circ}$ & .05 & .25 & 25.069 & 26.188 & 26.233 & 27.736 & 27.785 \\
\hline $5^{\circ}$ & .05 & .375 & 14.558 & 15.261 & 15.296 & 15.548 & 15.584 \\
\hline $5^{\circ}$ & .05 & .50 & 11.651 & 12.282 & 12.370 & 12.363 & 12.388 \\
\hline $10^{\circ}$ & .15 & .30 & 19.502 & 19.792 & 19.862 & 26.224 & 26.340 \\
\hline $10^{\circ}$ & .15 & .50 & 9.363 & 9.393 & 9.454 & 10.045 & 10.479 \\
\hline $10^{\circ}$ & .15 & 1.0 & 4.293 & 5.286 & 5.314 & 5.329 & 5.360 \\
\hline $15^{\circ}$ & .20 & .375 & 10.271 & 10.572 & 10.630 & 14.171 & 14.273 \\
\hline $15^{\circ}$ & .20 & 1.0 & 3.420 & 3.450 & 3.478 & 3.509 & 3.541 \\
\hline $20^{\circ}$ & .10 & .375 & 5.002 & 5.012 & 5.031 & 5.429 & 5.451 \\
\hline $20^{\circ}$ & .10 & .50 & 3.437 & 3.453 & 3.469 & 3.563 & 3.580 \\
\hline
\end{tabular}

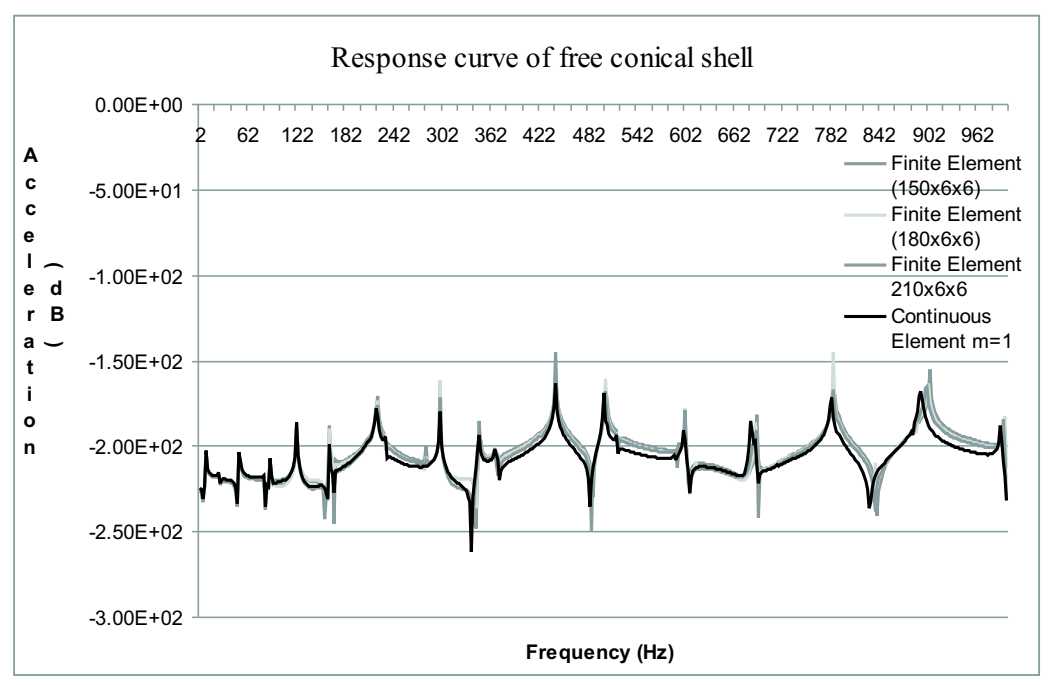

Fig. 6. Response curve of free conical shell 
The results are compared to those of Garnet and Kempner [5] who summarized some results based on different theories (Table 7). In general, values obtained by the method of continuous element are lower than those of other theories. Another observation is that the influence of the transverse deformation and rotary inertia becomes important when the conical is shortened. In general, there are no remarkable differences between our results and those of the literature.

\section{CONCLUSIONS}

A simple method to deal with the construction of the dynamic stiffness matrix of axisymmetric shells is presented. This method has been successfully used to develop a circular-basis cylindrical shell and a circular-basis conical shell continuous elements that take into account the rotatory inertia and shear deflection effects. Results are given in the case of cylindrical and conical shells for which it has been shown that the models that neglect the shear deformation and rotatory inertia are not suitable. Natural frequencies and the harmonic response obtained with this kind of formulation are in close agreement with finite element solutions. The main advantage is the reduction of the size of the model thus allows the high precision in the results for a large frequency range. The next research concerns the introduction of coupling effects with fluid or other kinds of structural elements such as laminated composite plates or shells.

\section{REFERENCES}

[1] Clough RW, Penzien J, Dynamic of Structures, New-York: McGraw-Hill, (1975).

[2] A. Kalnins, Analysis of shells of revolution subjected to symmetrical and nonsymmetrical loads, Journal of Applied Mechanics, 31(3) (1964), 467-476.

[3] Wilhelm Flüge, Stresses in Shells, Springer - Verlag - Berlin Heidelberg New York, (1973).

[4] Hervé Le Sourne, Devéloppement d"Eléments Continus de coques axisymetriques et de coudes, Thèse de Doctorat - Université de Nantes, (1998).

[5] Hyman Garnet and Joseph Kempner, Axisymmetric free vibrations of conical shells, Journal of Applied Mechanics, 31(3) (1964), 458-466.

[6] K.Y.Lam and Li Hua, Influence of boundary conditions on the frequency characteristics of a rotating truncated circular conical shell, Journal of Sound and Vibration, 143(2) (1998), 172-195.

[7] Casimir J. B., Nguyen Manh Cuong, Thick shells of revolution: Derivation of the dynamic stiffness matrix of continuous elements and application to a tested cylinder, Computers $\mathbb{E}$ structures,85(23-24) (2007), 1845-1857.

[8] Lunden R, Akesson B, Damped second order Rayleigh Timoshenko beam vibrationin space - an exact complex dynamic member stiffness matrix, Int J Numer Methods Eng, 19 (1983), 431-449.

[9] Leung AYT, Dynamic stiffness and substructures, New-York: Springer, (1993).

[10] Banerjee JR, Williams FW, Coupled bending-torsional dynamic stiffness matrix for Timoshenko beam elements, Comput Struct, 42 (1992), 301-310.

[11] Casimir JB, Duforet C, Vinh T, Dynamic behaviour of structures in large frequency range by continuous element methods, J. Sound Vib, 267 (2003), 1085-1106. 
[12] Casimir J B, Kevorkian S, Vinh T, The dynamic stiffness matrix of two-dimensional elements - application to Kirchhoff's plate continuous elements, J. Sound Vib, 287 (2005), 571-589.

[13] Nguyen Manh Cuong, Eléments Continus de plaques et coques avec prise en compte du cisaillement transverse, Application à l'interaction fluide-structure, Thèse de Doctorat, Université Paris VI (2003). 